\title{
Smart Digital Oscilloscope using Bluetooth and Android
}

\author{
Miss.Patil D.D. ${ }^{1}$, Prof.BhiseS.K. ${ }^{2}$ \\ Student, Department of E\&TC Engineering, DACOE, Karad, India ${ }^{1}$ \\ Assistant Professor, Department of E\&TC Engineering, DACOE, Karad, India ${ }^{2}$
}

\begin{abstract}
An oscilloscope, known as a scope, CRO or DSO is a type of electronic test instrument using which we can observe constantly time varying signal voltages, usually as a 2-D graph in which we can represent electrical voltages using the vertical axis plotted against time. Oscilloscopes nowadays we use today are CRO or DSO which is little bit difficult to use as well as not portable and consumes more power.

The system proposed consists of hardware device and an android application. This measuring System is based on Android device with application and equipped with Bluetooth Module which can measure input signal using Microcontroller and send this input signal to the Bluetooth of An Android Smartphone's. The hardware includes circuitry to capture the input voltage signals and an embedded Bluetooth module will transmit the captured signal information to an Android device for displaying the waveform. Using Android Smartphone we can observe Square Waveforms and Triangular Waveforms, Sinusoidal Waveforms. In this system an Android Software Application has been designed and developed for Smartphone based on android platform whose function is to display the information in the form of different parameters like Square, Triangular, and Sine Wave etc. This range for application to work is 30 meters between the Bluetooth of the Android device and external Bluetooth device. So this handy oscilloscope can be carried anywhere and is easy to use.
\end{abstract}

Keywords: dsPIC33FJ16GS504, Bluetooth module, Android phone.

\section{INTRODUCTION}

Oscilloscope is basic measurement device which is used In our project objective is:

for measuring purpose. It helps to you observe how the 1 . To implement a system to measure signals and display it voltage behave at any moment in time. In the CRO major on android phone.

disadvantage of CRO are to spend huge amount of money 2.To implement low cost, portable, low power to buy it and carry to difficult to carry from one place to consumption .the input will be processed by embedded another place. Now a day's portable Oscilloscope is system and it will transmitted Bluetooth module. available in the market nut they are very costly. In our project, we implement digital oscilloscope using Bluetooth and android system consisting of hardware device and software device application. New Technology, with low power consumption, dual Channel, portable. it is a Bluetooth embedded device which will capture input voltage signal and transmitted them to an external device Android is a Linux operating system design basically for touch screen mobile device such as a smart phone. Advantage of this android application is mainly environmental sustainable application, high mobility ,three time lesser than the cost of CRO and wireless the cathode ray oscilloscope is type of electronic test instrument that allow observation of constantly varying signal voltage, usually as a tow-dimensional graph of one or more electrical potential difference using the vertical or Y-axis, plotted as function as a time this allow the measurement of peak-to-peak voltage of a waveform, the frequency of periodic signal, the time between pulse, the time taken for a signal to rise to full amplitude, and relative timing of several related signal.

\section{LITERATURE SURVEY}

The development of an oscilloscope using Bluetooth was in earlier reported, it is prototype system called the "Android Bluetooth Oscilloscope", in which use Bluetooth module to send data to an Android phone which display the waveforms on its screen .where transmitter circuit uses Microchip dsPIC33FJ16GS504 and an LMX9838 Bluetooth 2.0 SPP module. [1]

In literature survey we found that the oscilloscopes they are currently available in the market are have some drawbacks: very costly, more power consumption and small resolve displays. Using PIC18F458 microcontroller bulky transmitter circuit for the analog-to-digital conversion of the input signals. The proceed data on the PIC are then transmitted to the phone via Bluetooth module. [2]

From research we found that data rate's upto 2 Mbps are not sufficient with existing software stacks designed on the PIC controller. Therefore, the approach recommended 
to fully utilize the bandwidth accessible by Bluetooth, was to use the module in Host controller Interface mode .using HCL mode,the onboard stack is removed and the module is placed in a state to executes the Bluetooth module bandwidth .hence the Bluetooth stack is not properly on the Bluetooth module, so it is necessary to be designed on the interfacing PIC processor. [3]

\section{III.Proposed SySTEM}

In our project system plan, it includes a Bluetooth module that is use for capture the sigma and transmits to the android device. This system is based on dsPIC33FI6GS504 controller fig 1 represent the block diagram of system.

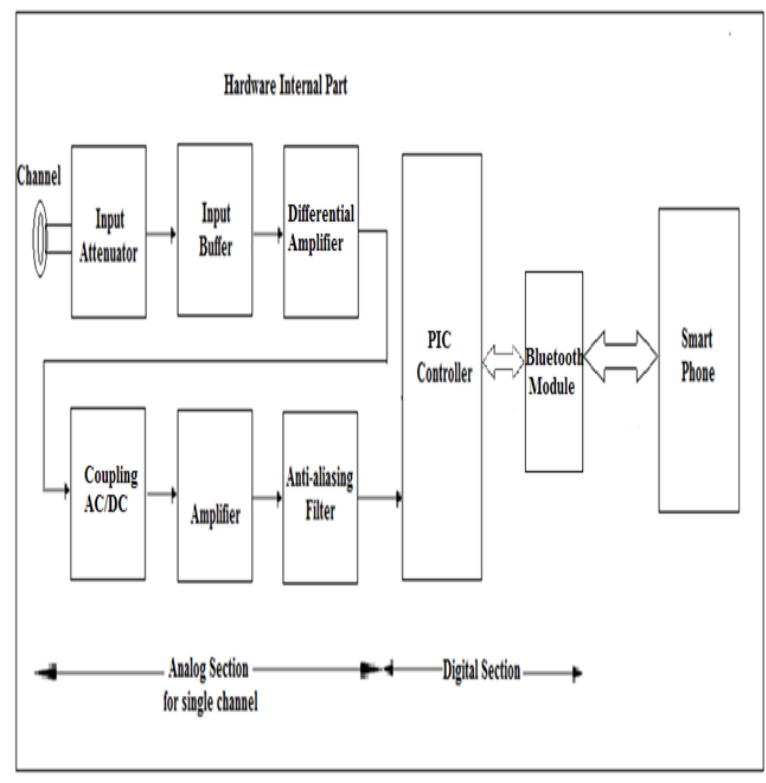

Fig.1.Block diagram

\section{A. PIC CONTROLLER:}

Today's world microcontroller is very important in every system. In our system microcontroller is use for sample the input signal and also use for calculating the floating point. In our system we use dsPIC33fj16gs504 PIC controller is used. It is high performance 16 bit digital signal controller within a small 44 pin QFN package. The DSPIC33FJ16GS504 is equipped with peripheral pin select which allow many of the digital peripheral to remapped to use any number of pins on the device. It has inbuilt ADC that converts a continuous physical quantity to a digital number that represents the quantity's amplitude.

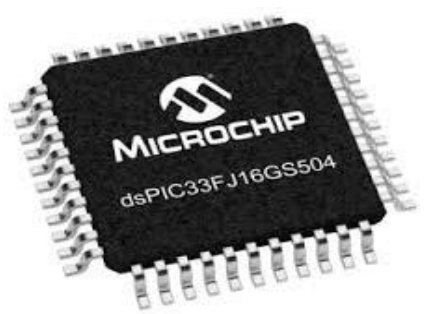

Fig.2 Microchip dSPIC33FJ16GS504

\section{B. BLUETOOTH MODULE:}

Bluetooth is wireless technology use for short range communication. This technology intended to replace the cable connecting portable or fixed device. It transmits data using low power radio waves. This technology has ability to handle both data and voice transmission simultaneously. Bluetooth technologies are robustness, low power, and low cost.

a) Frequency Spectrum: $2.45 \mathrm{GHz}$ (between $2.402 \mathrm{GHz}$ and $2.480 \mathrm{GHz})$.

b) Range: $10 \mathrm{~m}$ (32 Feet)

c) Data Rate: The version HC05 can support a data transmission rate of up to $3 \mathrm{Mbps}$.

d) Power: The commonly Bluetooth transmitter uses around $65 \mathrm{~mW}$ of power.

e) Cost: A USB Bluetooth HC05 adapter is available in the market for $\$ 10$.

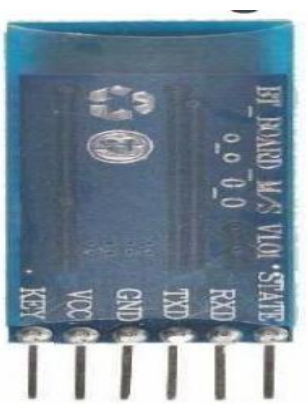

Fig.3 Bluetooth HC-05

\section{ANDROID SMART PHONE:}

Finally we observe the output of controller from Bluetooth module on android Smartphone. This is a touch screen device which will display output waveform on it.

D. INPUT ATTENUATOR:

An attenuator is an electronic device. It is used to reduce the power of a signal without distorting waveform. It has some key features are: Attenuation , Frequency bandwidth, power dissipation, SWR.

E. INPUT BUFFER:

A voltage amplifier is used to transfer a voltage from high input impedance level to low input impedance level. There are two types of buffer, voltage buffer and current buffer.

$F$. DIFFERNTIAL AMPLIFIER:

The differential amplifier is use to amplify the difference between two voltages.

G. COUPLING SELECTION:

Oscilloscope has two types of input coupling to handle alternating and direct current signals. When we set an input to DC coupling, the oscilloscope display both AC and DC signals and we set AC coupling, the oscilloscope display only AC signals.

1. AC Coupling:

AC coupling consist capacitor to filter out the DC signal component from $\mathrm{AC}$ and $\mathrm{DC}$ components.AC coupling blocks DC component and it allows AC component. The capacitor must be connected in series with the signal. Capacitive coupling is also known as AC coupling. 
IARJSET

International Advanced Research Journal in Science, Engineering and Technology

National Conference on Emerging trends in Electronics \& Telecommunication Engineering (NCETETE 2017) AGTI's Dr. Daulatrao Aher College Engineering, Vidyanagar Extension, Karad

Vol. 4, Special Issue 2, January 2017

\section{IV.FLOW CHART}

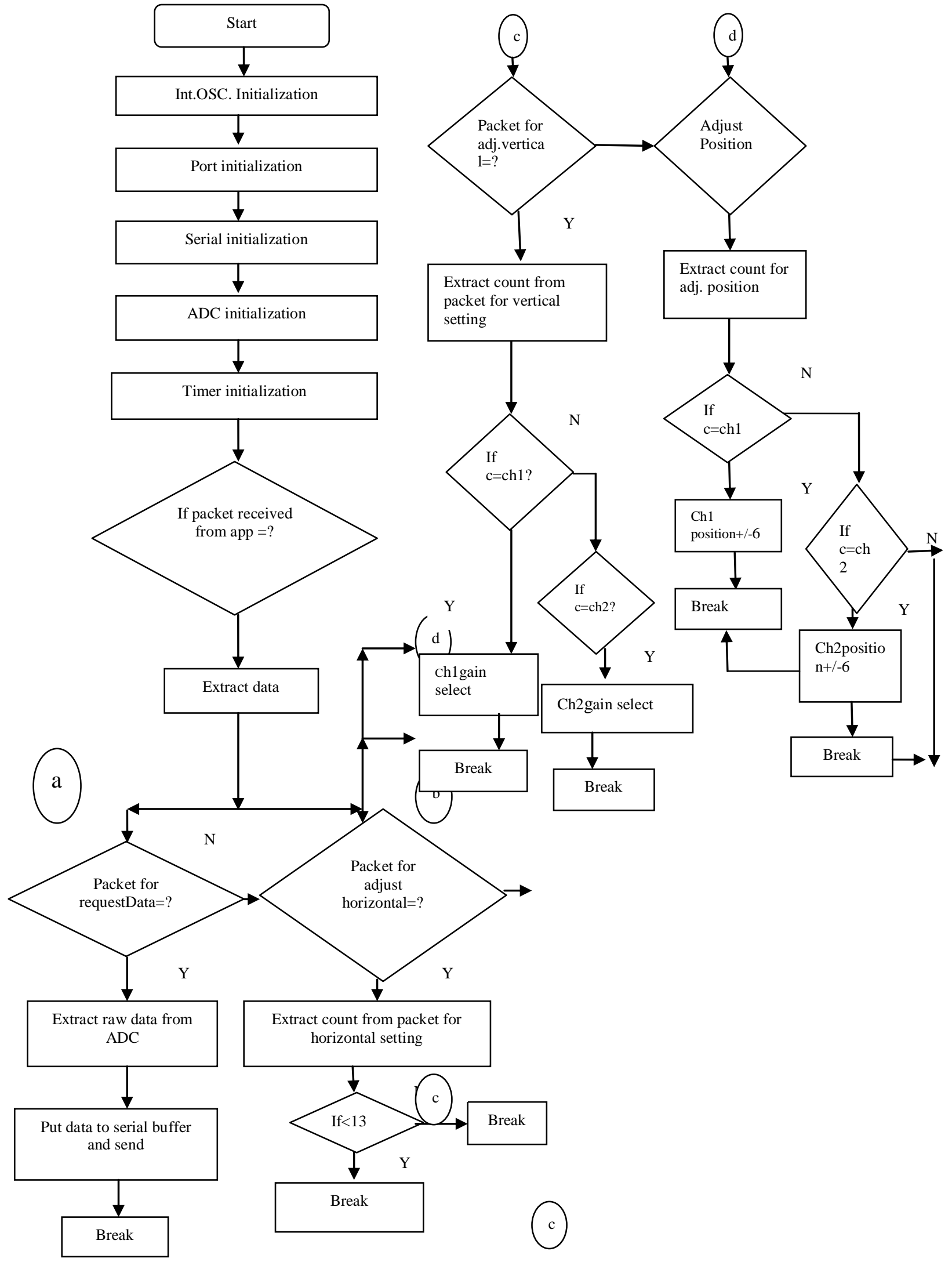




\section{DC Coupling:-}

The DC coupling setting provides a direct electrical path into the scope; it accepts all types of signals, including unchanging $\mathrm{DC}$ voltages, time-varying $\mathrm{DC}$ voltage, $\mathrm{AC}$, and combinations of $\mathrm{AC}$ and DC.

\section{H. ANTI-ALIASING FILTER:}

Before signal sampling anti-aliasing filter is used, to restrict the bandwidth of a signal to satisfy the sampling theorem. Filters are commonly used at the input of digital signal processing systems. The signal is sampled at a high rate, and then down sampled using a nearly ideal digital anti-aliasing filter.

\section{Conclusion}

This system represents a Bluetooth embedded device i.e. oscilloscope that selects optimum sampling rate and gain according to the setting selected. This system has the advantage that, the Android application can be upgraded to provide more features without doing changes to hardware, which helps to improve the device standard and user experience.

\section{REFERENCES}

[1] Yus “Android Bluetooth Oscilloscope"(2010 sep 23).

[2] MithilaJaybhey, Dipesh Kumar Pokar, Priyanka K. Katkar, Prof. A.A.Trikolikar "Wireless Oscilloscope using Zigbee" international journal of advanced research in computer engineering and technology, March2015.

[3] Prof.P.R.Badadapure,YogeshT.Vidhate,YuvrajR.Garud"Bluetooth Embedded Portable Oscilloscope powered by Android." international journal of advanced research in computer engineering and technology, March2015.

[4] KalyaniGanvir, HemantWaghmare , Oscilloscope on android phone, IRAJ International conference, 21 july, 2013.

[5] Prof.PrafullaP.Chaudhari,PratikPagare,SaurabhMeshram,AnkitPali wal "oscilloscope using Android". International journal of advanced research in Electronics and Communication engineering February 2016. 